\title{
STEM Analysis of Cycled Model Li -ion Battery Cathode Grown by MBE.
}

\author{
Bilash $\mathrm{KC}^{1}$, Jinglong $\mathrm{Guo}^{2}$, D. Bruce Buchholz ${ }^{3}$, Guennadi Evmenenko ${ }^{3}$ and Robert Klie ${ }^{1}$ \\ ${ }^{1}$ University of Illinois at Chicago, Chicago, Illinois, United States, ${ }^{2}$ University of Illinois at Chicago, \\ Cupertino, California, United States, ${ }^{3}$ Northwestern University, United States
}

Li-ion batteries (LIBs) are still the standard bearer for electrochemical energy storage technologies suitable for modern day portable electronics, electric vehicles, as well as large scale grid storage and other applications ${ }^{1,2}$. $\mathrm{LiMn}_{2} \mathrm{O}_{4}$ (LMO) cathode is a promising candidate for efficient intercalation of Li-ions at higher reversible concentrations. However, LMO cathodes still are vulnerable to capacity fading due to loss of $\mathrm{Mn}^{2+}$ ions during cycling and solid electrolyte interphase (SEI), which impedes mobility of Li ions resulting in low capacity retention ${ }^{1,2}$. Since electrolyte / electrode complex surface chemistry mostly determines the chemistry of the capacity fading mechanism, the in-depth analysis of surface phenomenon is critical.

LMO cathodes are generally studied in polycrystalline or nano-sized powders form, making detailed study of surfaces and interfaces difficult. To better understand surface chemistry single crystal cathodes with defined orientation is highly preferential. A considerable body of experimental evidence has shown that crystallinity, compositional homogeneity, crystallographic orientation, point defects, and grain boundaries influence Li ion transportation ${ }^{3,4}$ A novel method for single crystal epitaxial growth of LMO cathode is molecular beam epitaxy (MBE). Previously, we reported MBE grown single crystal thin film $\mathrm{LiMn}_{2} \mathrm{O}_{4}$ cathode on $\mathrm{SrTiO}_{3}$ substrate ${ }^{5}$. The unique feature of MBE grown LMO thin films is that it allows for precise control of compositional homogeneity, surface terminations, defect concentrations, crystal orientation, and stoichiometry which is fundamental while studying the surface reactions.

For electrochemistry of $\mathrm{LMO}$ thin films, a conductive buffer layer of $\mathrm{SrRuO}_{3}$ (SRO) of $10 \mathrm{~nm}$ thickness was deposited on $\mathrm{SrTiO}_{3}$ (STO), and $100 \mathrm{~nm} \mathrm{LiMn} \mathrm{O}_{4}$ thin film was grown on top using MBE. Figure 1(a) and 1(b) show the current collection setup for STO background and LMO thin film electrochemistry, respectively. Figure 1(c) shows the CV curves for the STO substrates and $\mathrm{LiMn}_{2} \mathrm{O}_{4} / \mathrm{SrRuO}_{3} / \mathrm{SrTiO}_{3}$. A pair of coupled anodic and cathodic peaks are seen for $\mathrm{Mn}^{3+} / \mathrm{Mn}^{4+}$ during charge/discharge corresponding to (de)intercalation of $\mathrm{Li}$ from $\mathrm{LiMn}_{2} \mathrm{O}_{4}$. For a slower scan rate of $0.1 \mathrm{mV} / \mathrm{s}$, the coupled redox peaks are present but are hard to distinguish due to the background noise as well as the dominant side reactions from electrolyte. However, for a higher scan rate of $1 \mathrm{mV} / \mathrm{s}$ the redox peaks are clearly visible and distinguishable. The measurements agree with the thin films measurements previously reported in the literatures ${ }^{6,7}$. The buffer layer and film both maintained good conductivity even at higher scan rate, which implies structural stability during the test conditions. The results show, for the first time, the cyclability of epitaxial $\mathrm{LiMn}_{2} \mathrm{O}_{4}$ thin film prepared on conductive SRO/STO by MBE. The atomic layer precision, and control over ratio of deposition elements provided by MBE means the opportunity to examine battery cathode thin films in both pristine and cycled states with control over film thickness, orientation, surface termination, passivating layers, doping concentrations etc. The good cyclability and stability of thin film even at higher scan rate could be beneficial for high power and fast charging applications.

Structural characterization using atomic-resolution STEM imaging and spectroscopies of the pristine and cycled films will be presented to quantify the changes in the surface structures and chemistry during the de(lithiation) process. Preliminary TEM examinations has shown that the buffer and film maintained epitaxial interfacial relationship and crystallinity. In our next step, we will examine the pristine film with the cycled film under different stages of charge/discharge cycle to observe the changes such as formation of SEI layer, surface phase transition from spinel to layered/rock salt structures, and capacity retention. 

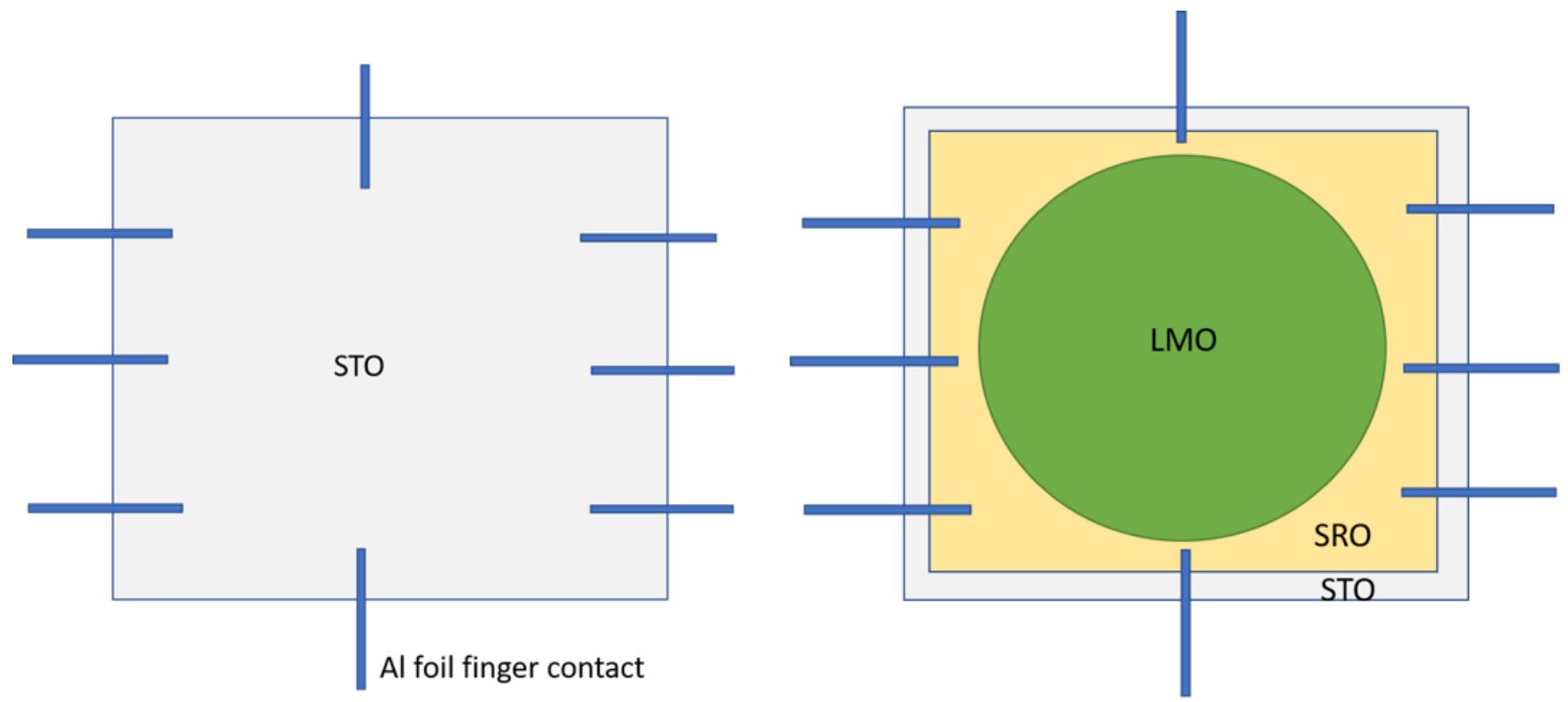

Figure 1. Electrochemistry set up for STO background and STO/SRO/STO film
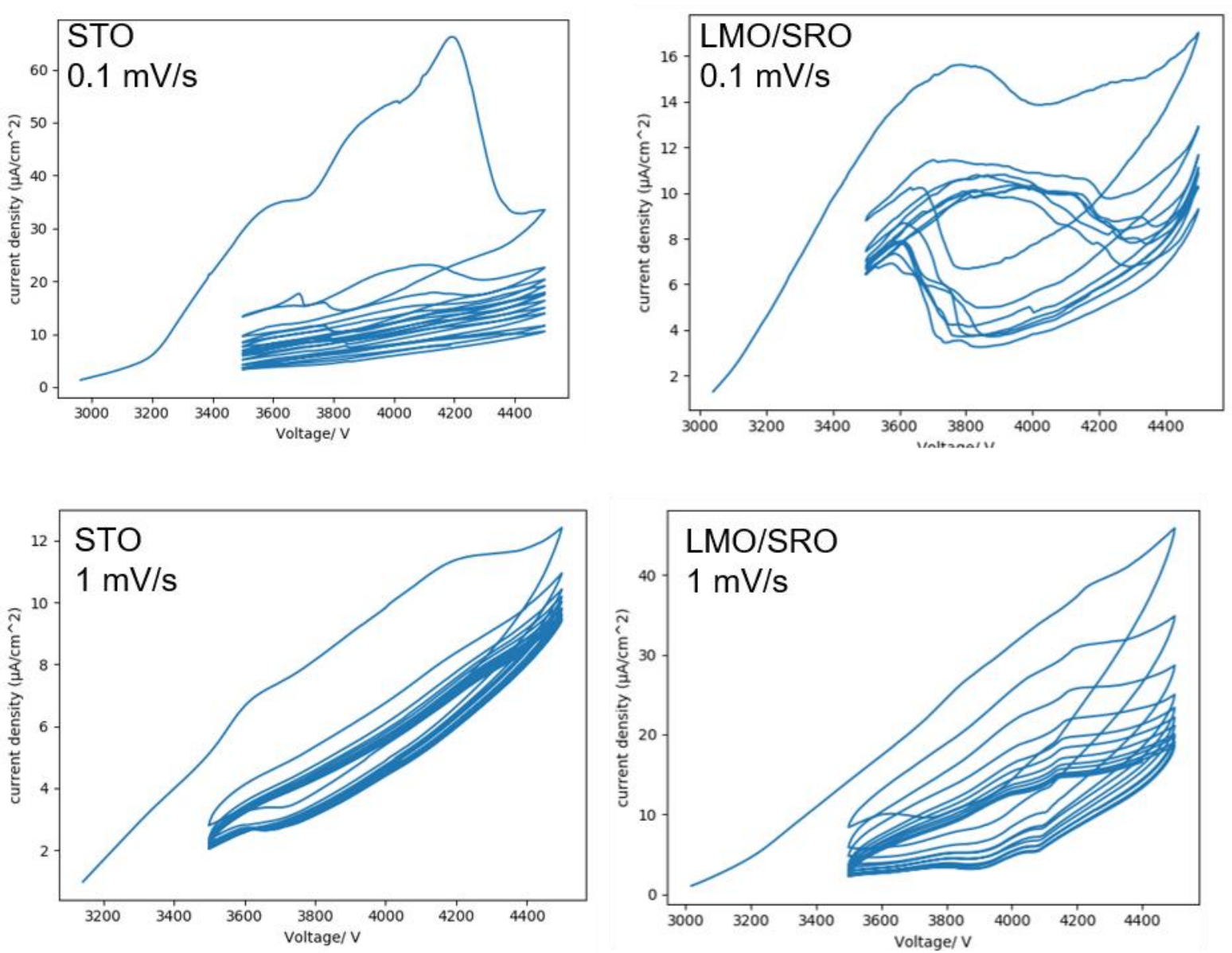

Figure 2. CV curves: STO background and LMO/SRO/STO 


\section{References}

[1] J. Zhao J \& Y. Wang, J. Phys. Chem. C 116 (2012), p. 11867-11876.

[2] J. Li et.al, ACS Appl. Mater. Interfaces 6 (2014), p. 18742-18750

[3] J. S. Kim et. al, Nano Lett. 12 (2012), p. 6358-6365.

[4] R. Yazami in "Nanomaterials for Lithium-Ion Batteries" ed. R.Yazami (CRC press, Florida) 261,269,277.

[5] B. KC et. al, Microscopy and Microanalysis 25.S2 (2019): p. 2086-2087.

[6] K. Suzuki et al, Journal of power sources 226 (2013), p. 340-345.

[7] B. Put et al, ACS Applied Materials \& Interfaces 7.40 (2015), p. 22413-22420. 